\title{
Chaotic pulsations in variable stars with harmonic mode coupling
}

\author{
Frank Verheest ${ }^{1}$ and Willy Hereman ${ }^{2}$ \\ ${ }^{1}$ Instituut voor theoretische mechanika, Rijksuniversiteit Gent \\ Krijgslaan 281, B-9000 Gent, Belgium \\ ${ }^{2}$ Department of Mathematical and Computer Sciences, Colorado School of Mines \\ Golden, CO 80401, U. S. A.
}

Some variable stars show multi-periodic behaviour with, among others, peaks in their power spectra at harmonically spaced frequencies with ratios 1:2:4. Such modes are nonlinearly coupled by two second-harmonic interactions and their amplitude equations are shown by a Painlevé analysis to be nonintegrable in a hamiltonian sense. Chaotic phenomena are thus expected, especially when other modes and dissipation are included. An example of stars to which this might apply is G191-16 among the variable white dwarfs.

\section{Introduction}

Some variable stars, such as certain white dwarfs, have individual pulsation amplitudes which vary with time. Power spectra of e.g. nonradial oscillations in certain ZZ Ceti stars contain, among others, prominent peaks at harmonically spaced frequencies with ratios 1:2:3, 1:2:4 or 1:2:3:4. Simultaneous nonlinear interactions between regularly spaced frequencies can theoretically be modelled by very special cases of mode coupling, in that selection rules combine three-mode coupling with degenerate cases of second harmonic generation (SHG), where two modes coalesce at half the frequency of another one. The nonlinear amplitude equations are fundamentally different from the usual three-mode or SHG equations, but can be brought into hamiltonian form for the regime without dissipation. Painlevé analysis shows that they are not integrable, in contrast to the simple three-mode or SHG cases. The 1:2:3 frequency spacing is covered elsewhere (Verheest, Hereman E Serras 1990), while the case 1:2:4 will be addressed here. Eventually, the system evolves chaotically, depending very sensitively upon initial conditions.

Before applying these findings to real stars, two inherent restrictions have to be discussed. First of all, other stellar modes have been left out, and secondly the analysis was done in a conservative framework. Real stars are dissipative, but the motivation for nevertheless concluding something about real stars is that if a simpler, hamiltonian model already points to chaotic behaviour, then the inclusion of additional modes and/or dissipation cannot improve matters (Verheest, Hereman $\&$ Serras 1990). Other researches reached similar conclusions concerning the possibility of chaotic pulsations, especially for the case of harmonic ratios 1:2:4 indicative of period-doubling phenomena, notably for the variable white dwarfs PG1351+489 (Goupil, Auvergne 83 Baglin 1988) and G191-16 (Vauclair et al. 1989). 


\section{Basic formalism}

Adhering to a model of three interacting modes, where the second mode is the second harmonic of the fundamental and the third mode is the second harmonic of the second, the combined selection rules for the angular frequencies $\omega_{j}(j=1,2,4)$ read

$$
\omega_{2}=2 \omega_{1}, \quad \omega_{4}=2 \omega_{2}=4 \omega_{1} .
$$

Consequently, the third mode is the fourth harmonic of the fundamental, hence the mnemonic use of the index 4 . The equations governing the slow time changes in the complex mode amplitudes $a_{j}$ are different from either the usual three-mode or the simple SHG cases, and also from the 1:2:3 frequency spacing case (Verheest, Hereman $\mathcal{E}$ Serras 1990), and are of the form (Verheest 1976)

$$
\dot{a}_{1}=2 i \lambda \bar{a}_{1} a_{2}, \quad \dot{a}_{2}=i \lambda a_{1}^{2}+2 i \mu \bar{a}_{2} a_{4}, \quad \dot{a}_{4}=i \mu a_{2}^{2},
$$

plus their complex conjugates. These equations are derivable from the Hamiltonian $H=\lambda\left(a_{1}^{2} \bar{a}_{2}+\bar{a}_{1}^{2} a_{2}\right)+\mu\left(a_{2}^{2} \bar{a}_{4}+\bar{a}_{2}^{2} a_{4}\right)$, in a description where complex conjugate variables are at the same time canonically conjugate (Verheest 1987). Besides the Hamiltonian, there is a second independent first integral $E=a_{1} \bar{a}_{1}+2 a_{2} \bar{a}_{2}+4 a_{4} \bar{a}_{4}$, a measure for the global mode energy, but that is not yet enough for complete integrability. Indications about integrability are given through a Painlevé analysis, see e.g. Menyuk, Chen $\&$ Lee (1983).

\section{Painlevé analysis}

We formally rewrite the system (2) as a set of ODEs in real variables $a_{j}$ and $A_{j}$ (coming from $\bar{a}_{j}$ ) by taking $\tau=i t$ as a new independent variable, hence

$$
\begin{array}{ll}
\dot{a}_{1}=2 \lambda A_{1} a_{2}, & \dot{A}_{1}=-2 \lambda a_{1} A_{2}, \\
\dot{a}_{2}=\lambda a_{1}^{2}+2 \mu A_{2} a_{4}, & \dot{A}_{2}=-\lambda A_{1}^{2}-2 \mu a_{2} A_{4}, \\
\dot{a}_{4}=\mu a_{2}^{2}, & \dot{A}_{4}=-\mu A_{2}^{2} .
\end{array}
$$

For the weak Painlevé test, we expand all variables as

$$
a_{j}=c_{j} \tau^{p_{j}}+d_{j} \tau^{p_{j}+r}+\cdots, \quad A_{j}=C_{j} \tau^{P_{j}}+D_{j} \tau^{P_{j}+r}+\cdots
$$

and try to find the most singular terms in each equation (with $c_{j} \neq 0$ and $C_{j} \neq 0$ ). Using (4) in (3) gives

$$
\begin{aligned}
p_{1} c_{1} \tau^{p_{1}-1} & =2 \lambda C_{1} c_{2} \tau^{P_{1}+p_{2}} \\
p_{2} c_{2} \tau^{p_{2}-1} & =\lambda c_{1}^{2} \tau^{2 p_{1}}+2 \mu C_{2} c_{4} \tau^{P_{2}+p_{4}}, \\
p_{4} c_{4} \tau^{p_{4}-1} & =\mu c_{2}^{2} \tau^{2 p_{2}} \\
P_{1} C_{1} \tau^{P_{1}-1} & =-2 \lambda c_{1} C_{2} \tau^{p_{1}+P_{2}} \\
P_{2} C_{2} \tau^{P_{2}-1} & =-\lambda C_{1}^{2} \tau^{2 P_{1}}-2 \mu c_{2} C_{4} \tau^{p_{2}+P_{4}} \\
P_{4} C_{4} \tau^{P_{4}-1} & =-\mu C_{2}^{2} \tau^{2 P_{2}}
\end{aligned}
$$

All equations, except the second and the fifth, are easy to balance and give

$$
\begin{array}{ll}
p_{2}=p-P-1, & P_{2}=-p+P-1, \\
p_{4}=2 p-2 P-1, & P_{4}=-2 p+2 P-1,
\end{array}
$$


if we call $p_{1}=p$ and $P_{1}=P$. Whether in the second and the fifth equation of (5) all terms are dominant or not (in the latter case $p+P+2>0$ is inferred), we find already useful results in the form

$$
c_{4}=\frac{\mu c_{2}^{2}}{2 p-2 P-1}, \quad C_{4}=\frac{\mu C_{2}^{2}}{2 p-2 P+1}, \quad c_{2} C_{2}=-\frac{p P}{4 \lambda^{2}} .
$$

In the case where $p+P+2>0$, we try to determine the values for $c_{j}$ and $C_{j}$ from (5) reduced to its most singular terms. We calculate that

$$
c_{2} C_{2}=\frac{(p-P+1)(2 p-2 P+1)}{2 \mu^{2}}=\frac{(p-P-1)(2 p-2 P-1)}{2 \mu^{2}}
$$

and have now three expressions for $c_{2} C_{2}$. The two expressions in (8) are only compatible provided $p=P$, in which case (7) and (8) yield

$$
c_{2} C_{2}=-\frac{p^{2}}{4 \lambda^{2}}=\frac{1}{2 \mu^{2}}
$$

There are thus no acceptable values for $p, \lambda$ or $\mu$, and we are led to the case where all terms are dominant, implying that $P=-p-2$. The values for $c_{j}$ and $C_{j}$ now have to be found from

$$
\begin{aligned}
p c_{1} & =2 \lambda C_{1} c_{2}, & (p+2) C_{1} & =2 \lambda c_{1} C_{2}, \\
(2 p+1) c_{2} & =\lambda c_{1}^{2}+2 \mu C_{2} c_{4}, & (2 p+3) C_{2} & =\lambda C_{1}^{2}+2 \mu c_{2} C_{4}, \\
(4 p+3) c_{4} & =\mu c_{2}^{2}, & (4 p+5) C_{4} & =\mu C_{2}^{2} .
\end{aligned}
$$

Combining some of these equations in a judicious way gives us two expressions for $c_{1} C_{1}$, namely

$$
c_{1} C_{1}=\frac{p}{2 \lambda^{2}}\left\{2 p+1-\xi \frac{p(p+2)}{4 p+3}\right\}=\frac{p+2}{2 \lambda^{2}}\left\{2 p+3-\xi \frac{p(p+2)}{4 p+5}\right\},
$$

having put for brevity $\xi=\mu^{2} / 2 \lambda^{2}$. Both expressions in (11) are only compatible if either $p=-1$, so that all the weights become equal to -1 , or

$$
p=-1 \pm \sqrt{\frac{1-\xi}{16-\xi}} \equiv-1 \pm q .
$$

This requires that $1-\xi$ and $16-\xi$ have the same sign, in other words that $0<\xi \leq 1$ or that $16<\xi$. We will return to this case further on, but first address the simpler case where all the weights are -1 . From (7), (10) and (11) we obtain

$$
\begin{aligned}
c_{2} & =-\frac{\lambda c_{1}^{2}}{1+\xi}, & C_{2}=\frac{\lambda C_{1}^{2}}{1+\xi}, & c_{1} C_{1}=\frac{1+\xi}{2 \lambda^{2}}, \\
c_{4} & =-\frac{\mu \lambda^{2} c_{1}^{4}}{(1+\xi)^{2}}, & C_{4} & =\frac{\mu \lambda^{2} C_{1}^{4}}{(1+\xi)^{2}} .
\end{aligned}
$$

We note that only one of the constants $c_{j}$ and $C_{j}$ can be taken arbitrary, either $c_{1}$ or $C_{1}$. The determination of the leading terms in (4) is thus complete and we move on to the next step in the Painlevé analysis, the determination of the resonances $r$. Keeping only the terms linear in $d_{j}$ and $D_{j}$ results in 


$$
\begin{array}{ll}
(1-r) d_{1}+2 \lambda\left(C_{1} d_{2}+c_{2} D_{1}\right) & =0, \\
(1-r) d_{2}+2 \lambda c_{1} d_{1}+2 \mu\left(C_{2} d_{4}+c_{4} D_{2}\right) & =0, \\
(1-r) d_{4}+2 \mu c_{2} d_{2} & =0, \\
(1-r) D_{1}-2 \lambda\left(c_{1} D_{2}+C_{2} d_{1}\right) & =0, \\
(1-r) D_{2}-2 \lambda C_{1} D_{1}-2 \mu\left(c_{2} D_{4}+C_{4} d_{2}\right) & =0, \\
(1-r) D_{4}-2 \mu C_{2} D_{2} & =0 .
\end{array}
$$

For this linear and homogeneous system in $d_{j}$ and $D_{j}$ to have a non-trivial solution we must equate the determinant of the coefficient matrix to zero. Using (13), the possible values for $r$ are then given by

$$
(r+1) r(r-2)(r-3)(r-1-\xi)(r-1+\xi)=0 .
$$

A resonance $r=-1$ corresponds to an arbitrary shift in the origin of $\tau$, and $r=0$ to an arbitrary constant $\left(c_{1}\right.$ or $\left.C_{1}\right)$ in the most singular terms. For the system to be integrable, the other resonances have to be non-negative integers and realisable, as we are expanding in ascending powers of $\tau$. This requires that

$$
1+\xi \geq 0, \quad 1-\xi \geq 0,
$$

therefore $\xi=1$ or $\xi=0$. A value $\xi=1$ would lead to two resonances zero, imposing that one could choose two of the $c_{j}$ and $C_{j}$ arbitrary, which cannot be done, however. The other possibility, $\xi=0$ or $\mu=0$, corresponds to simple SHG, long known to be integrable. Hence, we must conclude that the double SHG studied here is not integrable. Numerical computations have yielded positive Lyapunov exponents, even when initially all the energy is in the fundamental, indicating chaotic behaviour (see e.g. Steeb, Louw \& Villet 1987).

We return now to the case where $p=-1 \pm q$, so that (7), (10) and (11) give

$$
\begin{array}{ll}
c_{2}=-\frac{\lambda c_{1}^{2}}{2(1+q)}, & C_{2}=\frac{\lambda C_{1}^{2}}{2(1-q)}, \quad c_{1} C_{1}=\frac{1-q^{2}}{\lambda^{2}}, \\
c_{4}=\frac{\mu \lambda^{2} c_{1}^{4}}{4(4 q-1)(1+q)^{2}}, & C_{4}=\frac{\mu \lambda^{2} C_{1}^{4}}{4(4 q+1)(1-q)^{2}} .
\end{array}
$$

As in the previous case, where all the weights were -1 , only one of the constants $c_{j}$ and $C_{j}$ can be taken arbitrary and we arrive at the system

$$
\begin{array}{ll}
(1-r-q) d_{1}+2 \lambda\left(C_{1} d_{2}+c_{2} D_{1}\right) & =0, \\
(1-r-2 q) d_{2}+2 \lambda c_{1} d_{1}+2 \mu\left(C_{2} d_{4}+c_{4} D_{2}\right) & =0, \\
(1-r-4 q) d_{4}+2 \mu c_{2} d_{2} & =0, \\
(1-r+q) D_{1}-2 \lambda\left(c_{1} D_{2}+C_{2} d_{1}\right) & =0, \\
(1-r+2 q) D_{2}-2 \lambda C_{1} D_{1}-2 \mu\left(c_{2} D_{4}+C_{4} d_{2}\right) & =0, \\
(1-r+4 q) D_{4}-2 \mu C_{2} D_{2} & =0,
\end{array}
$$

for the $d_{j}$ and $D_{j}$. The resonances $r$ are obtained from

$$
(r+1) r(r-2)(r-3)\left(r-1-\sqrt{1+60 q^{2}}\right)\left(r-1+\sqrt{1+60 q^{2}}\right)=0,
$$

leading to nonintegrability on similar grounds as in the previous case. Because the system studied is not integrable, over long time periods we expect irregular 
phenomena, in sharp contrast to the usual periodic three-mode interactions. The presence of other modes would only increase the complexity and hence enforce the nonintegrability of the model, and so would dissipation.

\section{Chaotic pulsations}

Stars in which the power spectrum includes peaks at a fundamental frequency and its second and fourth harmonics (and hence for which our conclusions might be relevant) include certain ZZ Ceti stars. These are single, normal (hydrogen) DA white dwarfs with luminosity variations and hence denoted by DAV. There are also pulsating helium white dwarf (DBV) stars (see Winget (1988) for a review of these compact pulsators). The most pronounced of the relevant DAV stars is G191-16, with a light curve dominated by a frequency $\nu_{0}=1.12 \mathrm{mHz}$ and its harmonics at $2 \nu_{0}, 3 \nu_{0}$ and $4 \nu_{0}$ (Vauclair et al. 1989). Another example is the DBV star PG1351+489 with $\nu_{0}=1.028 \mathrm{mHz}$ (Goupil, Auvergne 83 Baglin 1988). Since both the special cases with frequency ratios 1:2:3 and 1:2:4 have now been shown to be nonintegrable, the general case with 1:2:3:4 spacing cannot be integrable either. Other $Z Z$ Ceti stars which include in their spectra the ratios 1:2:3:4 are $V Y$ Hor (= BPM31594) (with frequencies at 1.620, 3.240, 4.864 and $6.484 \mathrm{mHz}$ ) and its northern hemisphere twin $B G C V n=\mathrm{GD} 154$ (O'Donoghue 1986). The conclusions about deterministic low-order chaos can at this stage only be indicative, in view of the few stars studied so far observationally in any serious detail (Perdang 1990).

\section{Acknowledgments}

It is a pleasure for FV to thank the National Fund for Scientific Research (Belgium) for a research grant.

\section{References}

Goupil M J, Auvergne M and Baglin A 1988 Astron. Astrophys. 196, L13-L16

Menyuk C F, Chen H H and Lee Y C 1983 Phys. Rev. A 27, 1597-1611 \& J. Math. Phys. 24, 1073-1079

O'Donoghue D 1986 in: Seismology of the Sun and the Distant Stars (ed. D O Gough, Reidel, Dordrecht) 467-472

Perdang J 1990 in: Rapid variability of OB-stars: Nature and diagnostic value (in press)

Steeb W-H, Louw J A and Villet C M 1987 Aust. J. Phys. 40, 587-592

Vauclair G, Goupil M J, Baglin A, Auvergne M and Chevreton M 1989 Astron. Astrophys. 215, L17-L20

Verheest F 1976 Plasma Phys. 18, 225-234

Verheest F 1987 J. Phys. A: Math. Gen. 20, 103-110

Verheest F, Hereman W and Serras H 1990 Mon. Not. R. astron. Soc. 245, 392-396

Winget D E 1988 in: Advances in Helio- and Asteroseismology (eds. J ChristensenDalsgaard and S Fransen, Reidel, Dordrecht) 305-324 